\title{
Cosmological Model with Hybrid Expansion Law in Lyra Geometry
}

D.R.Manekar ${ }^{1 *}$, S. R. Bhoyar ${ }^{2}$, Hemant Kumar $^{3}$

\author{
${ }^{1}$ Research Scholar of Shri Jagdishprasad Jhabarmal Tibrewala University, Vidyanagri, Jhunjhunu, Rajasthan, India-333001 \\ ${ }^{2}$ Department of Mathematics, Phulsing Naik Mahavidyalaya, Pusad, Maharashtra- 445216 India \\ ${ }^{3}$ Department of Mathematics, Shri Jagdishprasad Jhabarmal Tibrewala University, Vidyanagri, Jhunjhunu, Rajasthan, India
}

DOI: $10.36347 /$ sipms.2021.v08i04.001

| Received: 15.03.2021 | Accepted: 19.04.2021 | Published: 24.04.2021

*Corresponding author: D.R.Manekar

\section{Abstract}

In this paper, we have studied anisotropic and homogeneous Bianchi type $\mathrm{V}$ universe filled with perfect fluid in the framework of Lyra's geometry. The solutions for field equations of cosmological models are obtained in Lyra's geometry by using two specific cases: firstly by taking the expansion scalar in the model is proportional to the shear scalar as and secondly by using the average scale factor as hybrid expansion form (combination of the power law and exponential form). Also, some Physical and Geometrical parameters are discussed in details.

Keywords: Bianchi type V universe, perfect fluid, Lyra's geometry.

Copyright $(\mathcal{C} 2021$ The Author(s): This is an open-access article distributed under the terms of the Creative Commons Attribution 4.0 International License (CC BY-NC 4.0) which permits unrestricted use, distribution, and reproduction in any medium for non-commercial use provided the original author and source are credited.

\section{INTRODUCTION}

In the frame work of general relativity, the acceleration in the expansion of the universe during recent cosmological times, first indicated by supernova observations [1] and also supported by the astrophysical data obtain from WMAP indicates the existence of an exotic fluid with negative pressure, which constitutes about the 70 percent of the total energy of the Universe.

Dark energy is a very useful concept since it encodes all our ignorance on the acceleration of the universe in a single cosmic component. Furthermore, Dark energy can also be used as an effective description of other mechanisms of the acceleration of the Universe [2].Several Candidates to present dark energy have been suggested with observations, Quintessence [3], Phantom [4], Brane-world Models [5], Pure Chaplygin gas model [6], Generalised Chaplygin Gas (GCG) model [7], modified Chaplygin Gas (MCG) model [8] and many others. Most recently, a new dark energy model, dubbed agegraphic darkenergy has been propose, which takes into account the Heisenberg Uncertainty relation of quantum mechanics together with the gravitational effect in general relativity. Because the holographic energy density belongs to adynamical cosmological constant, we need a dynamical frame to accommodate it instead of general relativity. Singh and Chaubey [9] have studied interacting dark energy in Bianchi type I space-time.
The viscous dark tachyon cosmology in interacting and non-interacting cases in non-flat FRW Universe was studied by Setare et al. [10]. Two-Fluid Cosmological Models in Bianchi Type-IIIand V Space-Time has been investigated by Adhav et al. $[11,12]$, Bianchi type-VI0 model with a two-fluid source has been investigated by Coley et al. [13]. Pant et al. [14] examined two fluid cosmological models using Bianchi type-II space-time. Two fluid Bianchi type-I models are studied by Oli [15]. Amirhashchi et al. [16, 17] have studied an interacting and non-interacting two-fluid scenario for dark energy models in FRW universe. Two-Fluid Cosmological Modelsin Bianchi Type-V Space-Time in Higher Dimensions examined by Mete et al. [18].

Several alternative theories of gravitation have been extensively discussed by many authors, to modify Einstein's general theory of relativity by incorporating certain desired features which are lacking in the original theory. One of the most intriguing modifications of general relativity is proposed by Weyl [19], invented to unify gravitation and electromagnetism by means of fundamental changes in Riemannian geometry. Unfortunately the Weyl theory suffers from non integrability of length which is physically unacceptable. However being interesting from mathematical point of view, it may still have the germs of a future fruitful theory. Later, Lyra [20] modified Riemannian geometry and removed non integrability of length transfer by introducing a gauge function into the structure-less 
manifold as a result of which a displacement field arises naturally. Subsequently, Sen et al. [21, 22] proposed a new scalar tensor theory of gravitation. They constructed an analog of the Einstein Field Equation based on Lyra's geometry. Halford [23, 24] showed that the scalar-tensor treatment based on Lyra's geometry predicts some effects within observational limitsas in Einstein's theory. Several authors Sri Ram and Singh [25] have obtained exact solutions of the field equations in vacuum and in the presence of stiff-matter for anisotropic Bianchi type $\mathrm{V}$ cosmological models in the normal gauge with a time-dependent displacement vector field. Singh [26] considered flat FRW model in Lyra's geometry by using varying adiabatic equation of state and solved the field equations for the early phases of evolution of universe. Pradhan and Vishwakarma [27] have investigated a class of LRS Bianchi type I models in the cosmological theory based on Lyra's geometry by considering a time-dependent displacement field for constant deceleration parameter of the Universe. Rahaman et al. [28] have obtained exact solutions for a spatially homogeneous and LRS Bianchi type-I model with constant deceleration parameter in Lyra's geometry. Kumar and Singh [29] have presented Bianchi type-I models in Lyra's geometry. Recently, Singh et al. [30] have obtained a new class of Bianchi type-I cosmological models in Lyra's geometry. Ram et al. [31] have obtained exact solutions for anisotropic Bianchi type $\mathrm{V}$ perfect fluid cosmological models in Lyra's geometry. Very recently, Chaubey [32] has obtained exact solutions for Kantowski-Sachs cosmological model in Lyra's geometry.

\section{Model and basic equations}

The diagonal form of the Bianchi type $\mathrm{V}$ metric is given by

$$
d s^{2}=d t^{2}-A^{2} d x^{2}-e^{2 m x}\left[B^{2} d y^{2}+C^{2} d z^{2}\right]
$$

Where the metric functions $A, B, C$ are functions of cosmic time $t$ and $m$ is a constant. A Bianchi type $\mathrm{V}$ model is a natural generalization of the open FRW model which eventually becomes isotropic. This model describes an anisotropic space-time and generates particular interest among physicists.

We first write some kinematical parameters of the metric such as the expressions for the average scale factor, volume scale factor, generalized Hubble's parameter, expansion scalar, the shear scalar and mean anisotropy parameter.

The average scale factor $a$ of the Bianchi type $\mathrm{V}$ model (1) is defined as

$$
a=(A B C)^{1 / 3}
$$

The special volume $V$ is given by

$V=a^{3}=A B C$.

We define the generalized Hubble's parameter $H$ as

$H=\frac{1}{3}\left(H_{1}+H_{2}+H_{3}\right)$

Where $H_{1}=\dot{A} / A, H_{2}=\dot{B} / B, H_{1}=\dot{C} / C$ are the directional Hubble's parameters in the directions of $x, y$ and $z$ respectively.
A dot denotes differentiation with respect to cosmic time $t$.

From equations (2)-(4), we obtain

$$
H=\frac{1}{3} \frac{\dot{V}}{V}=\frac{\dot{a}}{a}=\frac{1}{3}\left(\frac{\dot{A}}{A}+\frac{\dot{B}}{B}+\frac{\dot{C}}{C}\right) .
$$

Let us introduce the dynamical scalars such as the $\operatorname{expansion} \theta$, the shear scalar $\left(\sigma^{2}\right)$ and the mean anisotropy parameter $A_{m}$ as usual

$$
\begin{aligned}
& \theta=u_{; \mu}^{\mu} \\
& \sigma^{2}=\frac{1}{2} \sigma_{\mu v} \sigma^{\mu v} \\
& A_{m}=\frac{1}{3} \sum_{\mu=1}^{3}\left(\frac{\Delta H_{\mu}}{H}\right)^{2}
\end{aligned}
$$

Where $u^{\mu}=(0,0,0,1)$ is the matter 4-velocity vector. $\Delta H_{\mu}=H_{\mu}-H \cdot(\mu=1,2,3)$ and

$$
\sigma^{2}=\frac{1}{2}\left[\left(\frac{\dot{A}}{A}\right)^{2}+\left(\frac{\dot{B}}{B}\right)^{2}+\left(\frac{\dot{C}}{C}\right)^{2}\right]-\frac{\theta^{2}}{6} .
$$




\section{Field equations and their solutions}

The field equations in normal gauge for Lyra's geometry are

$$
R_{\mu \nu}-\frac{1}{2} g_{\mu \nu} R+\frac{3}{2}\left(\phi_{\mu} \phi_{\nu}-\frac{1}{2} g_{\mu \nu} \phi_{\alpha} \phi^{\alpha}\right)=-8 \pi G T_{\mu \nu}
$$

Where $\phi_{\mu}$ is the displacement vector defined as $\phi_{\mu}=(0,0,0, \beta(t))$, and $T_{\mu \nu}$ is the energy momentum tensor of the matter.

The energy momentum tensor $T_{\mu \nu}$ for a perfect fluid has the form

$$
T_{\mu v}=(\rho+p) u_{\mu} u_{v}-p g_{\mu v} \text {. }
$$

where $\rho$ is the energy density of the fluid, $p$ is the pressure, $u^{\mu}$ is the velocity vector satisfying $u_{\mu} u^{\mu}=1$.

In view of (11) for the Bianchi type V space-time (1), the field equation (10) lead to

$$
\begin{aligned}
& \frac{\ddot{B}}{B}+\frac{\ddot{C}}{C}+\frac{\dot{B}}{B} \frac{\dot{C}}{C}-\frac{m^{2}}{A^{2}}=-P-\frac{3}{4} \beta^{2} \\
& \frac{\ddot{A}}{A}+\frac{\ddot{C}}{C}+\frac{\dot{A}}{A} \frac{\dot{C}}{C}-\frac{m^{2}}{A^{2}}=-P-\frac{3}{4} \beta^{2} \\
& \frac{\ddot{A}}{A}+\frac{\ddot{B}}{B}+\frac{\dot{A}}{A} \frac{\dot{B}}{B}-\frac{m^{2}}{A^{2}}=-P-\frac{3}{4} \beta^{2} \\
& \frac{\dot{A}}{A} \frac{\dot{B}}{B}+\frac{\dot{A}}{A} \frac{\dot{C}}{C}+\frac{\dot{B}}{B} \frac{\dot{C}}{C}-\frac{3 m^{2}}{A^{2}}=\rho+\frac{3}{4} \beta^{2} \\
& \left(2 \frac{\dot{A}}{A}-\frac{\dot{B}}{B}-\frac{\dot{C}}{C}\right)=0 .
\end{aligned}
$$

We have assumed $8 \pi G=1$ in proper unit.

The energy conservation equation $T_{v ; \mu}^{\mu}=0$ takes the form

$$
\dot{\rho}+\frac{3}{2} \beta \dot{\beta}+\left[(\rho+P)+\frac{3}{2} \beta^{2}\right]\left(\frac{\dot{A}}{A}+\frac{\dot{B}}{B}+\frac{\dot{C}}{C}\right)=0 .
$$

For any physically relevant model, Hubble parameter (which provides the volumetric expansion rate of the Universe) and deceleration parameter (DP) (which tells whether the Universe exhibits accelerating volumetric expansion or not) are the most important observational quantities in cosmology. During 1960s and 1970s, red-shift magnitude test drew very categorical conclusions.

The DP lies between 0 and 1 , thus it was claimed that the Universe is decelerating [33]. Berman [34], Berman and Gomide [35] have proposed a law of variation for Hubble parameter that yields a power and exponential forms of the average scale factor with constant value of DP. In Berman's law, the DP $(q)$ can get value $1-\geq q$, and since $01 \leq \leq-q$ corresponds to accelerating expansion. So, many authors have studied cosmological models using this law but the recent theoretical analysis of type Ia supernovae (SNeIa)surveys large scale structure (LSS) and cosmic microwave background (CMB) anisotropy spectrum, which strongly indicate that our universe is spatially flat and has a phase transition i.e., past deceleration to recent acceleration. So, In order to match the results with this observation, many authors have defined different types of solutions (corresponds to DP and scale factor).

We consider the value of the average scale factor corresponding to the model of the Universe as

$a=t e^{t}$.

Using the equations (3) and (18) we have values of metric potentials

$$
\begin{aligned}
& A=t e^{t} \\
& B=\left(t e^{t}\right)^{2 n / n+1} \\
& C=\left(t e^{t}\right)^{2 / n+1}
\end{aligned}
$$

The metric (1) can be written as

$$
d s^{2}=d t^{2}-\left(t e^{t}\right)^{2} d x^{2}-e^{2 m x}\left\{\left[\left(t e^{t}\right)^{2 n / n+1}\right]^{2} d y^{2}+\left[\left(t e^{t}\right)^{2 / n+1}\right]^{2} d z^{2}\right\}
$$

\section{Some Physical and Geometrical Features of the model}

The physical parameters such as the Hubble parameter $H$, the anisotropic parameter $\left(A_{m}\right)$, the shear scalar $\left(\sigma^{2}\right)$, the expansion scalar $(\theta)$, and the spatial volume $V$ of model (25), which are of cosmological importance, are, respectively, given by 
$H=(1+1 / t)$

$\theta=3(1+1 / t)$

$A_{m}=2\left[\frac{n-1}{n+1}\right]^{2}$

$\sigma^{2}=3(1+1 / t)^{2}\left(\frac{n-1}{n+1}\right)^{2}$

$V=t^{3} e^{3 t}$

It is evident from the above result in equation (30) that the spatial volume is zero at $t=0$. But we observe that as cosmic time $t \rightarrow \infty$, the spatial volume expands to infinite. Therefore, the model starts evolving at $t=0$ and expands with cosmic time $t$. The mean anisotropy parameter is constant and different from zero for $n \neq 1$. From equation (28), it is observed that the mean anisotropy parameter of the present model becomes zero for $n=1$ and the anisotropy of the universe vanishes. But the universe is anisotropic throughout the evolution except for $n=1$. Other dynamical physical parameters such as expansion scalar $(\theta)$, shear scalar $\left(\sigma^{2}\right)$, and Hubble parameter $(H)$ diverge as cosmic time $t$ approaches to zero. These dynamical physical parameters are decreasing functions as cosmic time $t$ increases. Hence, the model (22) has a big bang type of initial singularity.

The deceleration parameter $(q)$ is obtained to be

$$
q=-\frac{a \ddot{a}}{\dot{a}^{2}}=-1+\frac{1}{(t+1)^{2}},
$$

Which is a constant value for late time throughout the evolution of the universe that means $q \rightarrow-1$ as $t \rightarrow \infty$. From equation (28), the universe will expand with decelerated rate for $q>0$ accelerated rate for $q<0$, and marginal inflation for $q=0$, One can explicitly observe the dependence of deceleration parameter $(q)$ on the constant parameters $n$.Thus, we can obtain a decelerated or accelerated expansion of the universe depending on the suitable choices of these parameters.

According to the recent observations of type Ia supernova (SNe Ia) [36-39] the present universe is accelerating and the value of deceleration parameter is in the range $-1<q<0$. Thus, the deceleration parameter of our model (22) is consistent with the recent astronomical observations.

By solving the equations (12) to (16), we get

The pressure of the model as,

$$
p=\frac{m^{2}}{t^{2} e^{2 t}}-\frac{3 / 4}{t^{6} e^{6 t}}-2\left(1+\frac{1}{t}\right)^{2}\left(1-\frac{2 n}{(n+1)^{2}}\right)-2\left(1+\frac{2}{t}\right)
$$

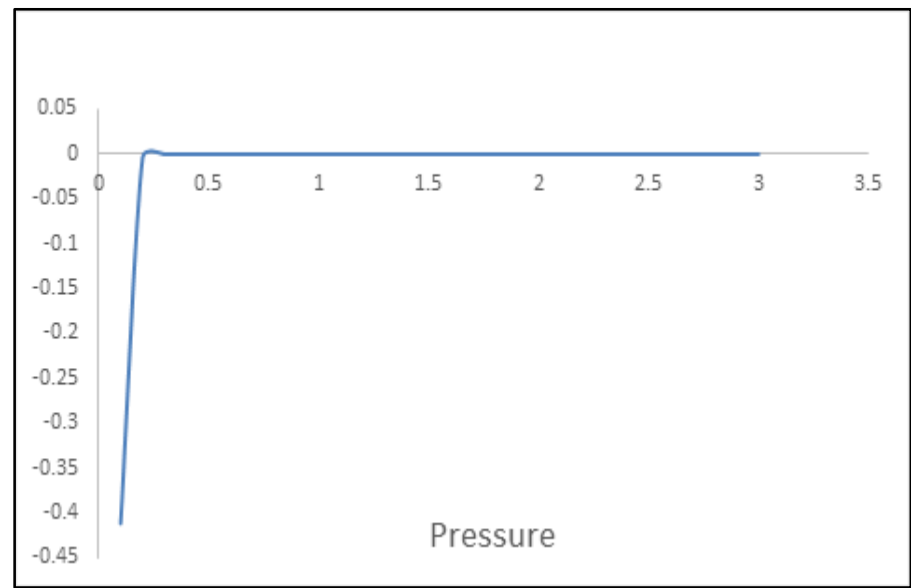

Fig-1: Pressure of the model versus time with the appropriate choice of constants.

Energy density of the model as,

$$
\rho=-\frac{3 / 4}{t^{6} e^{6 t}}-\left(1+\frac{1}{t}\right)^{2}\left(2+\frac{4 n}{(n+1)^{2}}\right)
$$




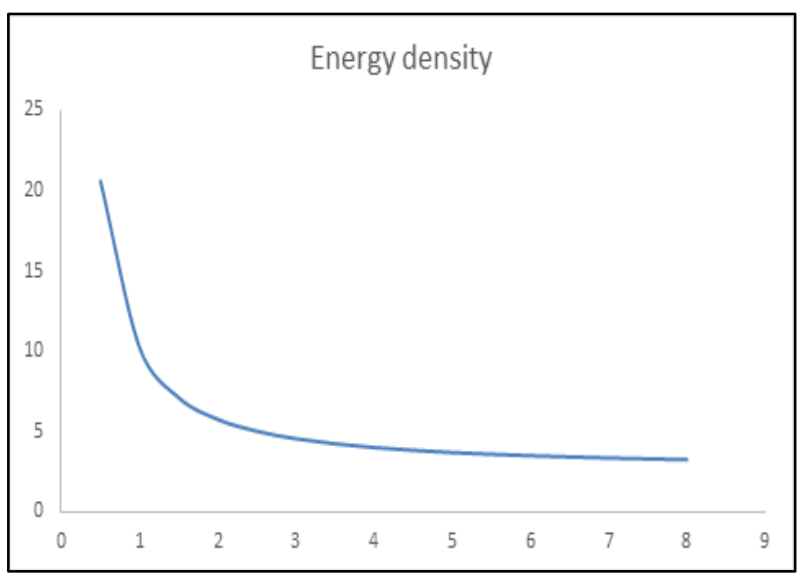

Fig-2: Energy density of the model versus time with the appropriate choice of constants

Gauge function of the model as,

$$
\beta=\frac{1}{t^{3} e^{3 t}}
$$

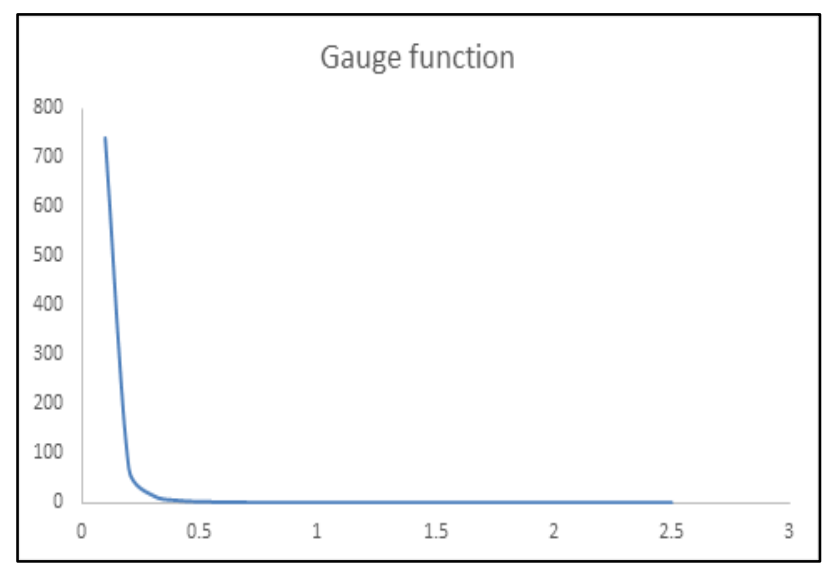

Fig-3: Gauge function of the model versus time with the appropriate choice of constants

From equation (31), we observe that at early time, the gauge function $\beta$ tends to infinity in this limit and also it tends to zero as cosmic time $t \rightarrow \infty$. It is large in the beginning and decreases fast with the evolution of the universe. Hence, $\beta$ is a decreasing function of cosmic time $t$. Thus, the model has singularity at $t=0$.It is clearly shows that the behavior of $\beta$ is as decreasing function of cosmic time t. It can be concluded that as $\mathrm{n}$ values increase, the values of $(\beta)$ decrease more with cosmic time increases. The physical parameters such as Hubble parameter $(H)$, anisotropic parameter $\left(A_{m}\right)$, shear $\operatorname{scalar}\left(\sigma^{2}\right)$, expansion $\operatorname{scalar}(\theta)$, spatial volume $(V)$, and deceleration parameter $(q)$ are found to be the same as in the above case of general relativity.

\section{CONCLUSIONS}

In this paper, we have studied anisotropic and homogeneous Bianchi type $\mathrm{V}$ universe filled with perfect fluid in the framework of Lyra's geometry. The solutions for field equations of cosmological models are obtained in Lyra's geometry by using two specific cases: firstly by taking the expansion scalar $(\theta)$ in the model is proportional to the shear scalar $\left(\sigma^{2}\right)$ as considered by Thorne [40] and Collins et al. [41] and secondly by using the average scale factor as hybrid expansion form (combination of the power law and exponential form). We have observed that the gauge function $(\beta)$ is a decreasing function of cosmic time $t$ in Lyra's geometry. The gauge function $(\beta)$ is large in the beginning and reduces fast with the evolution of the universe. It is found that for late cosmic times the gauge function $\beta \rightarrow 0$ and Lyra's geometry tends to general relativity in all respects. The present model of the gauge function $(\beta)$ is infinite at the initial singularity. The concept of the Lyra manifold is meaningful for finite time, but does not remain for very large time. But we have observed that the dynamical parameters: the average Hubble parameter, spatial volume, anisotropy parameter, expansion scalar and shear scalar, are the same in both frameworks. In each case, the cosmological models approach to anisotropic parameter for large value of cosmic time $t$. The model represents a 
D. R.Manekar et al., Sch J Phys Math Stat, Apr, 2021; 8(4): 82-87

shearing, nonrotating, and expanding universe, which approaches anisotropy for large value of time $t$. The present model is consistent with the recent observations of the present day of accelerating universe.

\section{REFERENCES}

1. Riess AG. 1998 Astron. J. 1161009 [SPIRES][astro-ph/9805201] Riess AG (Supernova Search Team Collaboration), 2004. Astrophys. J. 607:665.

2. Linder EV. Probing gravitation, dark energy, and acceleration. Physical Review D. 2004 Jul 28;70(2):023511.

3. Caldwell RR, Dave R, Steinhardt PJ. Cosmological imprint of an energy component with general equation of state. Physical Review Letters. 1998 Feb 23;80(8):1582.

4. Caldwell RR. A phantom menace? Cosmological consequences of a dark energy component with super-negative equation of state. Physics Letters B. 2002 Oct 3;545(1-2):23-9.

5. Alam UV. Sahni, astro-ph/ 0209443.

6. Kamenshchik A, Moschella U, Pasquier V. An alternative to quintessence. Physics Letters B. 2001 Jul 5;511(2-4):265-8.

7. Gorini V, Kamenshchik A, Moschella U. Can the Chaplygin gas be a plausible model for dark energy?. Physical Review D. 2003 Mar 26;67(6):063509.

8. Benaoum HB. Accelerated universe from modified Chaplygin gas and tachyonic fluid. arXiv preprint hep-th/0205140. 2002 May 14.

9. Singh T and Chaubey, R. RAA. 2012; 12, 473

10. Setare MR, Sadeghi J, Amani AR. Interacting tachyon dark energy in non-flat universe. Physics Letters B. 2009 Mar 30; 673(4-5):241-6.

11. Adhav KS. EJTP 8. 2011; 25, 319

12. Adhav KS. Int J TheorPhys.2011; 501846

13. Coley A, Dunn K. Two-fluid Bianchi VI (0) spacetimes. The Astrophysical Journal. 1990 Jan; 348:26-32.

14. Pant DN, Oli S. Two-fluid Bianchi type II cosmological models. Astrophysics and space science. 2002 Aug; 281(3):623-31.

15. Oli S. Astrophys. Space Sci. 2008; 314, 89

16. Amirhashchi H, Pradhan A, Saha B. Chin. Phys. Lett. 2011; 28, 039801

17. Amirhashchi H, Pradhan A, Zainuddin H. Int. J. Theor. Phys. 2011; 50, 3529

18. Mete VG. Prespacetime Journal, 3(2): 170

19. Weyl H. Sitz. ber. Preuss Akad. Wiss. 1918; 465.

20. Lyra G. Math. Z. 1951; 54, 52.

21. Sen DK, Phys Z. 1957; 149, 311.

22. Sen DK, Dunn KA. A scalar- tensor theory of gravitation in a modified Riemannian manifold. Journal of Mathematical Physics. 1971 Apr; 12(4):578-86.
23. Halford WD. Cosmological theory based on Lyra's geometry. Australian Journal of Physics. 1970;23(5):863-70.

24. Halford WD. Scalar- Tensor Theory of Gravitation in a Lyra Manifold. Journal of Mathematical Physics. 1972 Nov;13(11):1699-703.

25. Ram S, Singh P. Int. J. Theor. Phys. 1992; 31: 2095.

26. Singh CP. Astrophys. Space Sci. 2001; 275, 377.

27. Pradhan A, Vishwakarma AKJ. Geom. Phys. 2004; 49, 332

28. Rahaman F, Begum N, Bag G, Bhui BC. Cosmological models with negative constant deceleration parameter in Lyra geometry. Astrophysics and Space Science. 2005 Oct; 299(3):211-8.

29. Kumar S, Singh CP. An exact Bianchi type-I cosmological model in Lyra's manifold. International Journal of Modern Physics A. 2008 Mar 10; 23(06):813-22.

30. Singh GP, Kale AY. Int. J. Theor. Phys. 2009; 48, 2095

31. Ram S, Zeyauddin M, Singh CP. Anisotropic Bianchi type $\mathrm{V}$ perfect fluid cosmological models in Lyra's geometry. Journal of Geometry and Physics. 2010 Nov 1; 60(11):1671-80.

32. Chaubey R. Int. J. Theor. Phys. 2012. doi:10.1007/s10773-012-1285-5

33. Pradhan A, Shahi J and C. Singh, arXiv:grqc/0608070v2 (2006).

34. Berman MS. A special law of variation for Hubble's parameter. Il Nuovo Cimento B (19711996). 1983 Apr 1;74(2):182-6.

35. Berman M and Gomide F, Gen. Rel. Grav. 1988; 20, 191.

36. Perlmutter S, Aldering G, Goldhaber G, Knop RA, Nugent P, Castro PG, Deustua S, Fabbro S, Goobar A, Groom DE, Hook IM. Measurements of $\Omega$ and $\Lambda$ from 42 high-redshift supernovae. The Astrophysical Journal. 1999 Jun 1;517(2):565.

37. Riess AG, Strolge LG. J. Tonry, Astrophysical Journal. 2004; 607: 665-687.

38. Torny JL, Schmidt BP, Barris B. Astrophysical Journal. 2003; 594;1.

39. Clocchiatti A, Schmidt BP, Filippenko AV, Challis P, Coil AL, Covarrubias R, Diercks A, Garnavich P, Germany L, Gilliland R, Hogan C. Hubble Space Telescope and ground-based observations of type Ia Supernovae at redshift 0.5: cosmological implications. The Astrophysical Journal. 2006 May 1;642(1):1.

40. Thorne KS. Primordial element formation, primordial magnetic fields, and the isotropy of the universe. The Astrophysical Journal. 1967 Apr; 148:51.

41. Collins CB, Glass EN, Wilkinson DA. Exact spatially homogeneous cosmologies. General Relativity and Gravitation. 1980 Oct;12(10):80523. 